\title{
Digitales Lernen - Datenschutzrechtliche Rechtsgrundlagen von Lernplattformen für Kinder und Erwachsene
}

Maxi Nebel

\begin{abstract}
Lernplattformen bieten vielfältige Möglichkeiten des interaktiven, selbstbestimmten Lernens für Kinder und Erwachsene. Dabei gibt es eine große Bandbreite hinsichtlich der Systeme und deren Eigenschaften. Der folgende Beitrag untersucht, getrennt nach den verschiedenen Einsatzgebieten in der Schule einerseits und kommerzieller Angebote für Kinder und Erwachsene andererseits, welche Rechtsgrundlagen im jeweiligen Fall zum Einsatz kommen und welche datenschutzrechtlichen Anforderungen sich hieraus ergeben.
\end{abstract}

\section{Einleitung}

Ob virtuelles Klassenzimmer, Hausaufgaben, Nachhilfe, Ausbildung, berufsbegleitende Weiterbildung oder schlicht zur Freizeitgestaltung - webgestützte Lernplattformen bieten eine Reihe von Einsatzmöglichkeiten für alle Bevölkerungsgruppen. Dabei handelt es sich um Softwaresysteme zur Wissensvermittlung und Kommunikation zwischen Lernenden und Lehrenden. Sie bieten vielfältige Funktionalitäten: Neben dem Bereitstellen von Lerneinheiten, Arbeitsplänen, Arbeitsblättern, Tutorials und Videos bieten sie interaktive Übungen und kooperative Wikis sowie Kommunikationskanäle zwischen und unter Lehrenden und Lernenden.

Es gibt Softwarelösungen, die eigens für Schulen entworfen wurden und mithilfe derer jede Schule eine eigene Lernplattform nur für ihre Schülerinnen und Schüler ${ }^{1}$ betreiben kann. Bekanntes Beispiel hierfür ist die Software Moodle. Weiterhin gibt es allgemein zugängliche Lernplattformen, die von Verlagen, Softwareherstellern oder Medienanstalten be-

1 In diesem Beitrag wird aus Gründen der besseren Lesbarkeit im Folgenden das generische Maskulinum verwendet. Weibliche und anderweitige Geschlechteridentitäten werden dabei ausdrücklich mitgemeint. 
trieben werden. Deren Zielpublikum erstreckt sich von Kindern im Vorschulalter über Schüler bis hin zu spezifischen Angeboten im Bereich der Erwachsenenbildung. ${ }^{2}$

Mit der Nutzung solcher Lernplattformen geht eine umfangreiche Datenverarbeitung einher. Da Lernplattformen sowohl im Schulunterricht eingesetzt als auch durch privatwirtschaftliche Dienstleister angeboten werden, ist bei der datenschutzrechtlichen Beurteilung eine Differenzierung vorzunehmen. Plakativ ist dabei die Unterscheidung zwischen dem so genannten Vormittagsmarkt für die Verwendung von Lernplattformen in der Schule und dem Nachmittagsmarkt für die Verwendung von privatwirtschaftlich betriebenen, vielleicht auch kostenpflichtigen Lernplattformen, die außerhalb des Schulbetriebs von Kindern, aber auch von erwachsenen Lernenden genutzt werden. Der folgende Beitrag untersucht zunächst die Chancen und Risiken, die sich aus der Nutzung von Lernplattformen ergeben können. Anschließend werden die personenbezogenen Daten kategorisiert, die regelmäßig auf entsprechenden Plattformen erhoben werden, um sodann die zu deren Verarbeitung notwendigen Rechtsgrundlagen zu identifizieren. Rechtsgrundlagen entscheiden über die weiteren datenschutzrechtlichen Anforderungen, auf die aus Gründen des Umfangs in diesem Beitrag nicht im Detail eingegangen werden kann. Der Beitrag schließt mit technischen und organisatorischen Gestaltungsvorschlägen.

\section{Chancen und Risiken von Lernplattformen}

Lernplattformen bieten vielfältige Chancen für die Bildung von Menschen: Sie ermöglichen eine multimediale, interaktive und kooperative Lernumgebung sowie eine flexible Nutzung, ohne örtlich oder zeitliche gebunden zu sein. Durch sie können an die Bedürfnisse des Einzelnen angepasste Lernziele verfolgt und individuelle Lernfortschritte erfasst werden. Lerninhalte können gezielt auf die jeweilige Person ausgerichtet werden und sich somit an den Stärken und Schwächen eines jeden Lernenden orientieren. Interaktive Arbeitsformen ermöglichen eine Kooperation zwischen Lehrenden und Lernenden und zwischen Lernenden untereinander. ${ }^{3}$ Durch den Einsatz in Schulen wird die Medienkompetenz der Schüler von einem jungen Alter an gestärkt. Im Bereich der Erwachsenenbil-

2 Zum Datenschutz im E-Learning an Hochschulen s. Roßnagel 2020b: 296.

3 DSK 2018: $2 \mathrm{f}$. 
dung verstärkt die Niedrigschwelligkeit der Angebote (keine formalen $\mathrm{Zu}$ gangsvoraussetzungen, kostengünstige Programme) die Wahrscheinlichkeit, dass sich Lernende leichter zu allgemeinen oder berufsrelevanten Themen weiterbilden können (Life Long Learning).

Für die informationelle Selbstbestimmung und das Recht auf Datenschutz bergen Lernplattformen hingegen auch Risiken. Die umfassende Datenerhebung zu Art, Weise und Dauer der Nutzung, die Aufzeichnung des Lernfortschritts, -tempos und -inhalts verschafft einen Einblick in durchaus sensible Bereiche der persönlichen Fähigkeiten und Fertigkeiten und eröffnet damit die Möglichkeit, auf Charakter- und persönliche Eigenschaften rückzuschließen und Persönlichkeitsprofile zu erstellen. ${ }^{4}$

\section{Kategorisierung der personenbezogenen Daten auf Lernplattformen}

Bei der Nutzung von Lernplattformen werden eine Vielzahl verschiedener Daten verarbeitet. Diese lassen sich wie folgt kategorisieren. Notwendig sind zunächst der - möglichst pseudonyme - Benutzername und das Passwort. ${ }^{5}$ Handelt es sich jedoch um eine geschlossene Plattform, etwa die einer Schule, an der nur Schüler und Lehrkräfte dieser Schule teilnehmen dürfen, werden weitere Daten zur Identifizierung der Person benötigt. In diesem Fall ist der Klarname der betroffenen Person erforderlich. Bei Kostenpflicht des Dienstes sind weiterhin Zahlungsdaten erforderlich. Lernplattformen können überdies so gestaltet sein, dass der Nutzer ein eigenes personalisiertes Profil anlegen kann. Entsprechend der Ausgestaltung kann es Möglichkeiten geben, individuelle Angaben zur Person zu machen und ein Profilbild auf die Plattform zu laden. ${ }^{6} \mathrm{Da}$ sich privatwirtschaftlich betriebene Lernplattformen an alle Schulformen in allen Bundesländern richten, sind überdies häufig Angaben zum Bundesland, zum besuchten Schultyp und Jahrgangsstufe notwendig.

Sind die Nutzer von Lernplattformen Kinder, muss die Einwilligung des Sorgeberechtigten eingeholt werden. Da der Verantwortliche diese dokumentieren und die Identität des Sorgeberechtigten verifizieren muss, werden je nach Ausgestaltung der Dokumentation auch personenbezogene Daten der Sorgeberechtigten erhoben und mit dem Profil des Nutzers verknüpft.

4 DSK 2018: $3 \mathrm{f}$.

5 DSK 2018: 6 spricht von Stammdaten.

6 DSK 2018: 7 spricht von optionalen Daten. 
Weiterhin werden Daten zur Nutzung des Systems durch die betroffene Person erhoben. Zu diesen Nutzungsdaten gehören zum Beispiel IP-Adresse, An- und Abmeldezeit und damit Dauer der Nutzung, genutzte Dienste und anderes. ${ }^{7}$

Schließlich werden durch die Nutzung des Systems Daten mit inhaltlicher Relevanz zur Lernplattform erhoben. Man kann diese Kategorie als „Pädagogische Prozessdaten“ bezeichnen. ${ }^{8}$ Sie dienen dazu, dem Lehrenden den Lernprozess jedes einzelnen Nutzers oder einer ganzen Gruppe nachvollziehbar zu machen und anhand dessen das jeweilige Lernprogramm zu organisieren. Pädagogische Prozessdaten umfassen beispielsweise Kommentare und Nachrichten, Einträge in gemeinschaftlich bearbeiteten Datenbanken oder Dokumenten sowie Daten zur Bearbeitung von Lernobjekten, Aufgaben und Tests. Hinzukommen können weitere personenbezogene Daten durch zusätzlich implementierte Module (SCORM-, LTIModule, Live Classroom, Plagiatsüberprüfung), ${ }^{9}$ sofern der Verantwortliche entsprechende Funktionen in die Lernplattform eingebunden hat.

Weitere personenbezogene Daten können durch die Einbindung von Webanalyse-Diensten und Social Plug-ins entstehen. Webanalyse-Dienste sind spezielle Software-Tools, die Informationen zum Nutzungsverhalten, dem verwendeten Browser und dessen Einstellungen erheben. Sie werden eingesetzt, um beispielsweise Funktionalität der Website zu verbessern, zum Tracking der Nutzer und zum Einblenden personalisierter Werbung. Social Plug-ins sind Programmcodes, die Social Networks für Website-Betreiber zur Verfügung stellen und die diese in ihren Internetauftritt integrieren können. Bei Aufruf der Seite werden automatisch Daten an das Social Network geschickt, mindestens die aufgerufene URL und die IP-Adresse des Nutzers. ${ }^{10}$ Ist der Nutzer gegenüber dem Social Network authentifiziert, können ihm die erhobenen Daten zugeordnet werden. Betätigt der Nutzer das Social Plug-in, bekommt das Social Network die Möglichkeit, Cookies im Browser des Nutzers zu speichern. ${ }^{11}$ Mithilfe der Social Plugins wird es den Anbietern des Social Network erlaubt, über die Grenzen des Social Network hinaus Daten über Internetnutzer zu sammeln. Dies betrifft nicht nur eingeloggte Mitglieder, deren Daten mithilfe von Cookies direkt an das Social Network gesendet und ihrem Profil zugeordnet

7 DSK 2018: 8.

8 DSK 2018: $8 \mathrm{f}$.

9 DSK 2018: 9.

10 Z. B. Karg/Fahl 2011: 454; Jandt/Schaar/Schulz 2013: Rn. 117; Hornung 2015: Rn. 38. Zum Persönlichkeitsschutz in Social Networks ausführlich Nebel 2020.

11 Karg/Thomsen 2012: 731; Hornung 2015: Rn. 38. 
werden. Aber auch nicht eingeloggte Mitglieder sowie Nicht-Mitglieder können aufgrund von Social Plug-ins verfolgt und zu einem Profil zusammengeführt werden. ${ }^{12}$

\section{Rechtsgrundlagen für Schule als Verantwortliche}

Der Verantwortliche bedarf für die Datenverarbeitung auf seiner Lernplattform einer Rechtsgrundlage. Verantwortlicher ist nach Art. 4 Nr.7 DSGVO jede natürliche oder juristische Person, Behörde, Einrichtung oder andere Stelle, die allein oder gemeinsam mit anderen über die Zwecke und Mittel der Verarbeitung von personenbezogenen Daten entscheidet. Dies kann ein privatwirtschaftliches Unternehmen, eine Einzelperson, aber auch eine Schule sein. Für die Bestimmung der maßgeblichen Rechtsgrundlagen ist relevant, ob es sich um ein Unternehmen/eine Einzelperson oder um eine Behörde - bzw., da der Begriff Behörde funktional zu verstehen ist, ${ }^{13}$ jede öffentliche Anstalt - handelt. Diese werden daher im Folgenden getrennt betrachtet.

Die Datenschutz-Grundverordnung (DSGVO) ist auf Schulen sachlich anwendbar nach Art. 2 DSGVO. Der Ausschluss des Art. 2 Abs. 2 lit.a DSGVO greift nicht, wonach die DSGVO auf Bereiche keine Anwendung findet, die nicht im Anwendungsbereich des Unionsrechts liegen. Zwar ist Bildung Sache der Länder, die Union hat aber Kompetenz zu Unterstützungs-, Koordinierungs- und Ergänzungsmaßnahmen nach Art. 5 und 165 AEUV in Verbindung mit der Zuständigkeit im Rahmen des Datenschutzes nach Art. 16 AEUV. ${ }^{14}$ Sie ist auch örtlich anwendbar nach Art.3 DSGVO, weil und sobald die Schule nach Abs. 1 in der Union niedergelassen ist oder nach Abs. 2 lit. b, weil die Lernplattform der Beobachtung des Verhaltens der Lernenden dient.

Betreibt eine Schule eine Lernplattform, so kommen als Ermächtigungsgrundlage einzig die Erfüllung einer rechtlichen Verpflichtung nach Art. 6 Abs. 1 UAbs. 1 lit. c DSGVO sowie die Wahrnehmung von Aufgaben im öffentlichen Interesse nach lit. e in Betracht. Auf berechtigte Interessen nach lit. f kann sich eine öffentliche Anstalt gemäß UAbs. 2 ebenso wenig stützen wie auf eine wirksame Einwilligung.

12 Z. B. Karg/Fahl 2011: 454; Karg/Thomsen 2012: 731.

13 Schantz 2019: Rn. 97.

14 So auch Sassenberg 2019: Rn. 2. 


\subsection{Datenverarbeitung aufgrund gesetzlicher Ermächtigung}

Art. 6 Abs. 1 UAbs. 1 lit. c und e DSGVO können selbst keine Datenverarbeitung rechtfertigen, sondern bedürfen in Verbindung mit Abs. 2 und 3 der Rechtsgrundlage eines Mitgliedstaates. ${ }^{15}$ Ermöglicht ein Gesetz der Schule die Nutzung einer Lernplattform, kommt Art. 6 Abs. 1 UAbs. 1 lit. c DSGVO zur Anwendung. Die Nutzung einer Lernplattform im Rahmen des Unterrichts dient zudem der Unterrichtsgestaltung und Schülerausbildung. Sie liegt damit im öffentlichen Interesse nach Art. 6 Abs. 1 UAbs. 1 lit. e DSGVO. Die Datenverarbeitung muss erforderlich sein und es muss eine Rechtsgrundlage im mitgliedstaatlichen Recht vorliegen. Da das Schulwesen gemäß Art. 70 Abs. 1 in Verbindung mit Art. 72 ff. GG Sache der Länder ist, muss es sich bei dem mitgliedstaatlichen Recht um Landesrecht handeln, beispielsweise als Schuldatenschutz- oder Schulgesetz.

Im Hessischen Schulgesetz (HessSchulG) findet sich keine ausdrückliche Ermächtigung zum Einsatz von Lernplattformen. ${ }^{16}$ Daher ist zu prüfen, inwiefern allgemeine Regelungen anwendbar sind. Das Landesrecht muss im Wortlaut eindeutig sein, also die Nutzung einer interaktiven Plattform zur Gestaltung des Unterrichts ermöglichen. Formulierungen wie die datenschutzrechtliche Generalklausel in $\$ 83$ HessSchulG „zur Durchführung schulorganisatorischer Maßnahmen erforderlich“ ist nicht ausreichend, da sie nur das für die Verwaltung von Schulen Unabdingbare regelt $^{17}$ und schulorganisatorische Maßnahmen und der Unterrichtsauftrag auch durch weniger datenintensive Mittel erreicht werden können. Diese Annahme stützt auch die hessische Verordnung über die Verarbeitung personenbezogener Daten in Schulen und statistische Erhebungen an Schulen, ${ }^{18}$ die das Schulgesetz ergänzt und genau auflistet, welche personenbezogene Daten erhoben werden dürfen. Diese enthält jedenfalls keine Erlaubnis für Lernplattform-Daten.

Die Erlaubnis zur Datenverarbeitung ergibt sich damit beispielsweise für hessische Schulen aus dem Hessischen Datenschutz- und Informationsfreiheitsgesetz (HDSIG). Nach $\$ 3$ Abs. 1 HDSIG ist die Datenverarbeitung zulässig, wenn sie zur Erfüllung der in der Zuständigkeit der Schule lie-

15 Roßnagel 2019: Art. 6 Abs. 1 DSGVO, Rn. 52, 71; Schaller 2018: Rn. 8.

16 Anders hingegen z. B. $\$ 38$ b SächsSchulG.

17 Profit 2018: 3.

18 https://www.rv.hessenrecht.hessen.de/bshe/document/hevr-SchulStatErhVHEpAn lage1. 
genden Aufgabe oder in Ausübung öffentlicher Gewalt erforderlich ist. ${ }^{19}$ Der Bildungs- und Erziehungsauftrag der Schule ergibt sich aus $₫ 2$ HessSchulG. Diesen hat die Schule selbständig und in Eigenverantwortung umzusetzen, $\mathbb{S} \$ 127 \mathrm{ff}$. HessSchulG. Die Schulkonferenz beschließt im Rahmen ihrer gesetzlichen Befugnisse zum Entscheidungsrecht über das Schulprogramm die Bestimmung über den Einsatz einer Lernplattform an der jeweiligen Schule, $\mathbb{1} 129$ Nr. 1 in Verbindung mit $\$ 127 \mathrm{~b}$ HessSchulG. Beschließt sie den Einsatz einer Lernplattform, dürfen dafür erforderliche personenbezogene Daten nach $₫ 3$ Abs. 1 HDSIG verarbeitet werden. Für besondere Kategorien personenbezogener Daten gelten mangels Einschlägigkeit des $₫ 20$ HDSIG für Lernplattformen die Vorgaben des Art.9 DSGVO.

Der Einsatz einer Lernplattform kann grundsätzlich nicht auf eine Einwilligung nach Art. 6 Abs. 1 UAbs. 1 lit. a, Art. 7 DSGVO gestützt werden, da die Wirksamkeitsvoraussetzungen insbesondere der Freiwilligkeit nicht erfüllt werden können. ${ }^{20}$ Nach Erwägungsgrund 42 Satz 5 DSGVO ist eine Einwilligung etwa dann freiwillig, wenn die betroffene Person tatsächlich in der Lage ist, die Einwilligung zu verweigern oder zurückzuziehen, ohne Nachteile zu erleiden. Sie muss also eine echte Wahlmöglichkeit haben und keine Nachteile befürchten müssen. Die Freiwilligkeit der Einwilligung ist auch dann in Frage gestellt, wenn zwischen dem Verantwortlichen und der betroffenen Person ein klares Ungleichgewicht besteht. Beispielhaft für ein klares Ungleichgewicht ist nach Erwägungsgrund 43 DSGVO das Verhältnis von Behörden zu betroffenen Personen. Für die Tätigkeiten von Behörden stellt die DSGVO dafür gesonderte Ermächtigungstatbestände und Öffnungsklauseln für eigene Regelungen der Mitgliedstaaten auf. Da zwischen Schule und Schüler ein Über-/Unterordnungsverhältnis besteht, dem sich der Schüler durch die bestehende Schulpflicht nicht entziehen kann, ist eine freiwillige Entscheidung unmöglich. Wird der Einsatz einer Lernplattform in der Klasse von der Einwilligung aller Schüler abhängig gemacht, kann sich der einzelne Schüler nicht entziehen, ohne Nachteile befürchten zu müssen. Dennoch wird beispielsweise in Sachsen-Anhalt beim Einsatz von Lernplattformen eine Einwilligung der Schüler bzw. der Eltern gefordert. ${ }^{21}$ Dies ist, wie dargelegt, unrechtmäBig.

19 Für Hochschulen s. Roßnagel 2020b: 296.

20 Anders hingegen in Hochschulen, s. Roßnagel 2020b: 296.

21 Vgl. Vereinbarung über die Bereitstellung und Nutzung einer Instanz der Lernplattform Moodle für Schulen in Sachsen-Anhalt 2018, https://www.bildung-lsa.d 
Mittels Lernplattformen können auch automatisierte Einzelentscheidungen wie Versetzungsentscheidungen, Leistungsbewertungen, Kurseinstufungen oder ähnliches gefällt werden. Die Zulässigkeit von automatisierten Einzelentscheidungen richtet sich nach Art. 22 DSGVO. Art. 22 Abs. 1 DSGVO gibt der betroffenen Person das Recht, nicht einer ausschließlich auf einer automatisierten Verarbeitung beruhenden Entscheidung unterworfen zu werden, die ihr gegenüber rechtliche Wirkung entfaltet oder sie in ähnlicher Weise beeinträchtigt. Die automatisierte Generierung von Einzelentscheidungen - oder vielmehr: algorithmenbasierte Entscheidung im Einzelfall - ist nach Abs. 2 lit. b und c dann zulässig, wenn unions- oder mitgliedstaatliches Recht sie erlaubt oder die betroffene Person eingewilligt hat. Im Schulrecht obliegen diese Entscheidungen ausschließlich den zuständigen Lehrkräften und der Klassenkonferenz und sind damit nach Art. 22 Abs. 2 DSGVO nicht zulässig. ${ }^{22}$

Die sonstigen Voraussetzungen der DSGVO sowie spezifischer landesgesetzlicher Regelungen - soweit vorhanden - sind einzuhalten. $\mathrm{Zu}$ beachten ist aber, dass in den landesgesetzlichen Regelungen gemäß Art. 6 Abs. 2 DSGVO spezifischere Bestimmungen zur Anpassung der Anwendung der Vorschriften der DSGVO in Bezug auf die Verarbeitung zur Erfüllung von Absatz 1 Buchstaben c und e beibehalten oder eingeführt werden können, sofern sie die Vorgaben der DSGVO präzisieren und konkretisieren. ${ }^{23}$

Der Verantwortliche hat also vor allem Informationspflichten einzuhalten, Betroffenenrechte zu gewährleisten und die Vorgaben zum Datenschutz durch Technik umzusetzen. Eine Datenschutz-Folgenabschätzung nach Art. 35 DSGVO ist notwendig, wenn die Datenverarbeitung ein hohes Risiko für die betroffenen Personen zur Folge hat. Da der Einsatz von Lernplattformen nur aufgrund einer gesetzlichen Grundlage möglich ist, ist eine Datenschutz-Folgenabschätzung gemäß Abs. 10 vorzunehmen, wenn nicht bereits eine allgemeine Folgenabschätzung im Zusammenhang mit dem Erlass der Rechtsgrundlage erfolgte und dies nach Einschätzung des zuständigen Gesetzgebers nötig ist. ${ }^{24}$

e/files/b91568b2b16749b6bd47d9d05ff2c859/LISA_Nutzungsvereinbarung_Schul instanz.pdf.

22 In Hessen ergeben sich die Befugnisse zu Versetzungsentscheidungen, Leistungsbewertungen und Kurseinstufungen aus $₫ 73$ Abs. 3, $₫ 75$ Abs. 4, $\mathbb{7} 76$ Abs. 2 HessSchulG.

23 Zum Regelungsumfang des Art. 6 Abs. 2 DSGVO Roßnagel 2019: Art. 6 Abs. 2 DSGVO, Rn. $22 \mathrm{ff}$.

24 Ausführlich zum Verfahren nach Art.35 Abs.10 DSGVO Roßnagel/Geminn/ Johannes 2019: 435. 


\subsection{Social Media an Schulen}

Eine gesonderte Betrachtung verdient der Einsatz von Social Media an Schulen. WhatsApp wird regelmäßig zur Kommunikation unter Schülern sowie zwischen Schülern und Lehrern genutzt. Video-Plattformen wie YouTube fungieren bei vielen Kindern und Jugendlichen als Online-Lehrer. ${ }^{25}$ Auch andere Social Media-Kanäle eignen sich nicht nur zum Kommunizieren, sondern auch zum Lernen, Recherchieren und Analysieren. Ein gezielter Einsatz von Social Media im Unterricht hätte für Schulen den Vorteil, Medienkompetenz quasi nebenbei zu vermitteln, und zwar auf Plattformen, die Kinder ohnehin nutzen.

Die Regelungen der einzelnen Bundesländer reichen - sofern sie sich überhaupt zu dem Thema positionieren - von einem ausdrücklichen Nutzungsverbot bis hin zum erwünschten Einsatz für Lehrzwecke. Das Bremische Schuldatenschutzgesetz erlaubt in $\$ 4$ Abs. 4 den Einsatz von Social Media, wenn dies dem Schulleben dient, die spezifische Plattform der DSGVO entspricht und die Schulleitung eingewilligt hat. In Baden-Württemberg untersagt das Kultusministerium etwa den Einsatz von Social Media zur Kommunikation zwischen Lehrkräften und Schülern, verweist aber stattdessen auf Lernplattformen wie Moodle. Nach einer Handreichung des hessischen Kultusministeriums sollte „[d]ie Nutzung von öffentlichen oder kommerziellen Sozialen Netzwerken im Bereich der schulischen und unterrichtsrelevanten Kommunikation (...), wenn überhaupt, nur sehr eingeschränkt erfolgen. Das Hessische Kultusministerium empfiehlt vielmehr die Nutzung von schulinternen Lernplattformen, wie beispielsweise Schul-Moodle Hessen (...).“26

Aus datenschutzrechtlicher Sicht sprechen viele Gründe gegen den Einsatz von Social Media. ${ }^{27}$ Wenn ein Schulgesetz oder eine Schule den Gebrauch von Social Media im Unterricht vorschreibt, benötigt jedes Kind ein entsprechendes Nutzerkonto, da eine Nutzung ohne eigenes Konto regelmäßig nicht möglich ist. Die Voraussetzungen der Einwilligung nach Art. 7 und 8 DSGVO müssen auch hier beachtet werden. Allerdings kann von einer freiwilligen Einwilligung kaum die Rede sein. Bei der datenschutzrechtlichen Beurteilung bleiben viele Punkte problematisch, etwa zur Frage der Verantwortlichkeit für die Datenverarbeitung. Ob die Haus-

25 Ratzsch 2019.

26 https://datenschutz.hessen.de/datenschutz/hochschulen-schulen-und-archive/nutz ung-sozialer-netzwerke-durch-lehrkräfte.

27 Zum Persönlichkeitsschutz in Social Networks ausführlich Nebel 2020. 
haltsausnahme des Art. 2 Abs. 2 lit. c DSGVO greift, muss bezweifelt werden, da im schulischen Kontext schwerlich von einer ausschließlich privaten und familiären Nutzung ausgegangen werden kann. ${ }^{28}$ Da jeder Nutzer, also auch jedes Kind, personenbezogene Daten anderer Nutzer verarbeitet und für diese dann datenschutzrechtlich verantwortlich ist, treffen diesen auch die Pflichten zum Einholen einer Einwilligung und zur Gewährleistung der Betroffenenrechte. Problematisch ist außerdem die Datenübertragung in Drittstaaten, wenn das Unternehmen - wie Facebook oder Google - seinen Sitz nicht in der Union hat. Setzt eine Schule Social Media ein, gilt es alles dies zu bedenken, da datenschutzrechtliche Pflichten von der Schule auf die Nutzer abgewälzt würden, wenn der Einsatz von Social Media vorgeschrieben wäre.

Ebenso problematisch ist die eigenständige Nutzung von Social Media durch die Schule. Bindet eine Schule das Social Network in ihre eigene Kommunikationsstruktur ein, indem sie eine öffentliche Profilseite bei einem Social Network - etwa Fanpages bei Facebook oder einen Videokanal bei YouTube - für ihren Öffentlichkeitsauftritt betreibt oder um Informationen an ihre Schüler zu vermitteln, stellt sich die Frage, inwiefern sie für die dabei verarbeiteten personenbezogenen Daten der Nutzer verantwortlich ist. Der Europäische Gerichtshof hat sich zur Frage der Verantwortlichkeit von Fanpage-Betreibern eindeutig geäußert. Die Verarbeitung personenbezogener Daten ermöglicht Facebook nicht nur, das werbebasierte Geschäftsmodell zu verbessern. Es ermöglicht darüber hinaus den Betreibern der Fanpage, Kenntnis über die Profile der Besucher zu erlangen. Damit verfolgt der Fanpage-Betreiber eigene Zwecke und trägt durch das Einrichten der Fanpage maßgeblich dazu bei, dass personenbezogene Daten der Besucher durch Facebook erhoben werden. ${ }^{29}$ Dadurch ist der Fanpage-Betreiber ebenso gemäß Art. 4 Nr. 7 DSGVO Verantwortlicher für die Datenverarbeitung, und zusammen mit dem Anbieter sind beide gemeinsam im Sinne des Art. 26 DSGVO für die Verarbeitung verantwortlich. ${ }^{30}$ Die Schule muss also gemäß Art. 26 Abs. 1 DSGVO eine Vereinbarung mit dem Social Network treffen, wer welche Verpflichtungen aus der DSGVO, insbesondere hinsichtlich der Informationspflichten und Gewährleistung der Betroffenenrechte, zu erfüllen hat. Aus diesen Gründen ist es grundsätzlich nicht empfehlenswert, als Schule eine öffentliche Profilseite oder einen Videokanal bei einem Social Network zu betreiben.

28 Ausführlich Roßnagel 2019: Art. 2 DSGVO, Rn. $23 \mathrm{ff}$.

29 EuGH, Urt. v. 5.6.2018, Rs. C-210/16, ECLI:EU:C:2018:388, Rn. 34-36 - Fanpage.

30 EuGH, Urt. v. 5.6.2018, Rs. C-210/16, ECLI:EU:C:2018:388, Rn. 39, 43 - Fanpage. 


\subsection{Altersgrenze zur Geltendmachung von Betroffenenrechten}

Als Verantwortliche hat die Schule die Betroffenenrechte der Schüler zu gewährleisten. Diese ergeben sich in erster Linie aus Art. 15 ff. DSGVO. Im Rahmen des Art. 23 DSGVO können Mitgliedstaaten durch eigene Rechtsvorschriften von den Vorgaben der DSGVO abweichen. Ist die Schule für die Datenverarbeitung verantwortlich, sind zur Bestimmung der Ausgestaltung der Betroffenenrechte auch die Landesvorschriften, in Hessen beispielsweise $\mathbb{S} 31 \mathrm{ff}$. HDSIG, zu beachten. Ab wann Kinder als betroffene Personen ihre Betroffenenrechte selbst wahrnehmen dürfen, ist gesetzlich nicht festgelegt. Da die DSGVO keine Regelungen hierzu trifft, richtet sich die Beurteilung der Altersgrenze nach der Einsichtsfähigkeit des jeweiligen Kindes und bedarf damit einer Einzelfallentscheidung. Der Landesgesetzgeber darf grundsätzlich eine Typisierung nach dem Alter der Schüler vornehmen, um einheitliche Regelungen zu erlassen und nicht in jedem Einzelfall nach der Einsichtsfähigkeit entscheiden zu müssen. Da dies einen Eingriff in die informationelle Selbstbestimmung der Schüler darstellt, muss ein entsprechendes Gesetz angemessen und erforderlich sein. Besser noch wäre ein gemeinsamer Standpunkt der Kultusministerkonferenz, um gleichmäßige Altersgrenzen in allen Bundesländern zu etablieren. Dies ist bisher nicht geschehen. ${ }^{31}$

\section{Rechtsgrundlage für sonstige Betreiber als Verantwortliche}

Lernplattformen werden nicht nur von Schulen betrieben, sondern beispielsweise auch von privatwirtschaftlichen Unternehmen oder Verlagen. Die DSGVO ist anwendbar nach Art. 3 DSGVO, wenn der Verantwortliche nach Abs. 1 in der Union niedergelassen ist oder nach Abs. 2 lit. a, wenn er der betroffenen Person Waren oder Dienstleistungen, also etwa elektronische Lernplattformen, anbietet. Diese dienen auch der Beobachtung des Verhaltens, so dass die DSGVO auch nach Abs. 2 lit. b Anwendung findet. Als Rechtsgrundlage kommt die Einwilligung nach Art. 6

31 Einzig der Freistaat Sachsen hat eine Vorschrift erlassen, und in Abschnitt IV Nr. 2 der sächsische Verwaltungsvorschrift Schuldatenschutz eine Altersgrenze von 14 Jahren zur selbständigen Geltendmachung von Betroffenenrechten festgelegt. Die Regelung ist inhaltlich nicht zu beanstanden, allerdings formell rechtswidrig, da es sich nicht um ein parlamentarisches Gesetz handelt. 
Abs. 1 UAbs. 1 lit. a DSGVO in Frage, die Verarbeitung zur Vertragserfüllung nach lit. $\mathrm{b}$ sowie berechtigte Interessen nach lit. f.

Die Systematik des Art. 6 Abs. 1 DSGVO sieht vor, dass grundsätzlich mehrere Erlaubnistatbestände einschlägig sein und alternativ herangezogen werden können („mindestens“). Gerade im Verhältnis von Einwilligung nach lit. a und berechtigtem Interesse nach lit.f kommt es aber zu Spannungen zwischen dem Interesse des Verantwortlichen und der Erwartungshaltung der betroffenen Person hinsichtlich der Kontrolle über ihre personenbezogenen Daten. ${ }^{32}$ Der Verantwortliche muss sich vor der Datenverarbeitung für den jeweiligen Zweck verbindlich auf die konkrete Rechtsgrundlage festlegen und die betroffene Person darüber entsprechend informieren, sonst widerspräche er dem Grundsatz von Treu und Glauben nach Art. 5 Abs. 1 lit. a DSGVO, wenn er sich nachträglich auf eine andere Rechtsgrundlage berufen würde.

\subsection{Verarbeitung aufgrund eines Vertrags}

Nach Art. 6 Abs. 1 UAbs. 1 lit. b DSGVO ist eine Verarbeitung rechtmäßig, wenn sie zur Erfüllung eines Vertrags oder zur Durchführung vorvertraglicher Maßnahmen, die auf Anfrage der betroffenen Person erfolgen, erforderlich ist. Erforderlich ist die Datenverarbeitung, wenn sie zur Erfüllung von Pflichten aus dem Vertrag benötigt werden. ${ }^{33}$ Die vertragscharakteristische Leistung bei Lernplattformen ist die Bereitstellung von Alter und besuchtem Schulzweig der betroffenen Person abhängigen Lerninhalten sowie gegebenenfalls andere Leistungen wie Videoübertragung oder Möglichkeiten zur Plagiatsprüfung. ${ }^{34}$ Erforderlich sind Stammdaten zum Einloggen auf die Plattform (Nutzername und Passwort) sowie Angaben zur Jahrgangsstufe, Schulzweig sowie Bundesland des Wohnortes. Auch die pädagogischen Prozessdaten lassen sich über lit. b rechtfertigen, da sie zur Erfüllung der Aufgaben der Lehrpersonen und damit zur Erbringung der Dienstleistung erforderlich sind. Erforderlich sind außerdem etwa die IPAdresse und Session-Cookies, die während der Sitzung zur Aufrechterhaltung des Dienstes benötigt werden. ${ }^{35}$

32 Dazu Schantz 2019: Rn. 88 ff. sowie Nebel 2020: 178 f.

33 Z. B. Schantz 2019: Rn. 24 ff., Nebel 2020: 198 ff.

34 Vgl. DSK 2018: 9.

35 So auch Schulz 2018: Art. 6 DSGVO, Rn. 34. 
Art. 6 Abs. 1 UAbs. 1 lit. b DSGVO setzt voraus, dass die betroffene Person Vertragspartei ist. Ist die betroffene Person volljährig, ist dies unproblematisch. Im Falle von Lernplattformen für Kinder heißt das aber, dass der Verantwortliche einen wirksamen Vertrag mit dem Kind abschließen muss. Das Kind muss nach den Regeln des $\mathbb{S} 104 \mathrm{ff}$. BGB vom Sorgeberechtigten vertreten werden. Da der Abschluss eines Dienstleistungsvertrags - in der Regel bereits aufgrund der Zahlungspflicht - nicht ausschließlich rechtlich vorteilhaft ist, muss der Sorgeberechtigte gemäß $\$ 107$ BGB einwilligen. In der Praxis werden auf Lernplattformen regelmäßig die Eltern angesprochen, einen Vertrag abzuschließen, damit deren Kind die Plattform nutzen kann. Der Wortlaut der DSGVO ist in dieser Hinsicht eindeutig: Es bedarf einer entsprechenden Ausgestaltung des Vertrags, in denen die Eltern nicht als eigener Vertragspartner, sondern nur in Vertretung des betroffenen Kindes handeln.

\subsection{Verarbeitung aufgrund berechtigter Interessen}

Kann sich der Verantwortliche aus rechtlichen Gründen nicht auf Art. 6 Abs. 1 lit. b DSGVO stützen, wird er ein berechtigtes Interesse an der Verarbeitung der Stamm- und pädagogischen Prozessdaten geltend machen. Eine Datenverarbeitung ist nach Art. 6 Abs. 1 UAbs. 1 lit. f DSGVO zulässig, wenn die Verarbeitung zur Wahrung berechtigter Interessen des Verantwortlichen oder eines Dritten erforderlich ist, und sofern nicht die Interessen oder Grundrechte und Grundfreiheiten der betroffenen Person, die den Schutz personenbezogener Daten erfordern, überwiegen. Der Verantwortliche ist verpflichtet, eine umfassende Interessenabwägung vorzunehmen und zu dokumentieren. Lit. f legt aber fest, dass die Interessen oder Grundrechte und Grundfreiheiten in der Regel überwiegen, wenn die betroffene Person ein Kind ist, denn die Schutzbedürftigkeit der Kinder, insbesondere derer unter 16 Jahren, gebietet es, die Eltern in die Entscheidung über die Datenverarbeitung mit einzubinden, ${ }^{36}$, also deren Einwilligung einzuholen. Daher kann lit. f in der Regel nicht für die Rechtfertigung der Verarbeitung der Stamm- und pädagogischen Prozessdaten der Kinder herangezogen werden.

Häufig wird der Verantwortliche als berechtigtes Interesse zudem die Verarbeitung personenbezogener Daten zu Werbezwecken geltend machen. Nach Erwägungsgrund 47 DSGVO „kann“ Direktwerbung als be-

36 Buchner/Petri 2018: Rn. 155. 
rechtigtes Interesse betrachtet werden. Dies schließt personalisierte Werbung grundsätzlich mit ein. ${ }^{37}$ Diese ist jedoch wesentlich eingriffsintensiver als herkömmliche Werbung, da hierfür ein umfassendes Persönlichkeitsprofil erstellt wird. Je umfassender das Persönlichkeitsprofil der betroffenen Person, desto eher überwiegen ihre Interessen, Grundrechte und Grundfreiheiten dem Werbeinteresse des Anbieters. Zu berücksichtigen ist auch die Möglichkeit der betroffenen Person zur Wahrnehmung ihrer Betroffenenrechte. So ist etwa das Recht auf Datenübertragbarkeit aus Art. 20 DSGVO nur bei einer Einwilligung als Rechtsgrundlage möglich, nicht hingegen bei einer Interessenabwägung. Datenverarbeitung zum Zwecke personalisierter Werbung wird daher nicht über lit. f gerechtfertigt werden können, erst recht nicht, wenn es sich bei der betroffenen Person um ein Kind handelt. Auch wenn die Sorgeberechtigten die Vertragspartner der Plattformbetreiber sind, so werden die Plattformen doch durch die Kinder genutzt und diese sind damit auch die betroffenen Personen, deren Verhalten getrackt wird und denen personenbezogene Werbung präsentiert wird. Dieser „Missverhältnis“ spricht noch mehr dafür, in jedem Fall eine ausdrückliche Einwilligung einzuholen.

Im Ergebnis kann eine Datenverarbeitung für Werbezwecke nicht über Art. 6 Abs. 1 lit.f DSGVO gerechtfertigt werden. Gleiches gilt für die personenbezogenen Daten von Kindern, die Lernplattform nutzen. Vielmehr bedarf es in beiden Fällen einer Einwilligung.

\subsection{Datenverarbeitung aufgrund einer Einwilligung}

Kann sich der Verantwortliche nicht auf die Vertragsdurchführung oder berechtigte Interessen berufen, muss er eine Einwilligung nach Art. 6 Abs. 1 UAbs. 1 lit. a DSGVO in Verbindung mit Art. 7 DSGVO einholen. Die Voraussetzungen der Einwilligung ergeben sich aus einem Zusammenspiel von Art. 4 Nr. 11 mit Art. 6 Abs. 1 UAbs. 1 lit. a sowie Art. 7 DSGVO $^{38}$ und werden zusätzlich durch die allgemeinen Verarbeitungsgrundsätze des Art. 5 DSGVO ergänzt. Es müssen also alle Voraussetzungen einer wirksamen Einwilligung vorliegen. Handelt das Kind als betroffene Person selbst, gilt zusätzlich die Regelung des Art. 8 DSGVO.

37 Z. B. Schantz/Wolff 2017: Rn. 666, Gierschmann 2018: 9 ff.; Nebel 2020: 206 ff., Helfrich 2020: Rn. 77. A. A. Schulz 2018: Art. 21 DSGVO, Rn. 20.

38 Kritisch zum Regelungskonzept Heckmann/Paschke 2018: Art. 7 DSGVO, Rn. 2. 
Gemäß Art. 4 Nr. 11 DSGVO handelt es sich bei der Einwilligung um eine unmissverständlich abgegebene Willensbekundung ,in Form einer Erklärung oder einer sonstigen eindeutigen bestätigenden Handlung“. Die „eindeutige bestätigende Handlung“ besteht nach Erwägungsgrund 32 DSGVO zum Beispiel in einer schriftlichen, elektronischen oder mündlichen „Erklärung“, mit der die betroffene Person ihr Einverständnis mit der Datenverarbeitung signalisiert. Diese Erklärung kann auch durch das Anklicken eines Kästchens, durch „Auswahl technischer Einstellungen für Dienste der Informationsgesellschaft" oder andere Verhaltensweisen abgegeben werden. Stillschweigen, Inaktivität und vorangekreuzte Kästchen sind nach Erwägungsgrund 32 DSGVO ebenso wenig ausreichend wie die schlichte Inanspruchnahme eines Dienstes. ${ }^{39}$ Die DSGVO fordert somit ein Opt-in in die Datenverarbeitung. Die Opt-out-Lösung des Bundesgerichtshofs ${ }^{40}$ ist nach der DSGVO nicht mehr zulässig. ${ }^{41}$

Die Willensbekundung muss „in informierter Weise“ abgegeben worden sein. Die betroffene Person muss die Möglichkeit gehabt haben, den Inhalt der Erklärung zur Kenntnis nehmen ${ }^{42}$ und Auswirkungen, Umstände und Tragweite der Datenverarbeitung erkennen zu können. ${ }^{43}$ Die Einwilligung ist für einen oder mehrere bestimmte eindeutige und legitime Zwecke zu geben, sie muss demnach bestimmt genug sein. Der Zweck der Datenverarbeitung muss vom Verantwortlichen zum Zeitpunkt der Einwilligung so präzise festgelegt werden, dass die betroffene Person in der Lage ist zu beurteilen, aus welchem Grund der Verantwortliche bestimmte personenbezogene Daten verarbeiten möchte.

Schließlich muss die Einwilligung freiwillig erteilt worden sein. Eine Einwilligung ist freiwillig, wenn sie auf der freien Entscheidung der betroffenen Person beruht und ohne Zwang gegeben wurde. Die betroffene Person muss also eine echte Wahl haben. ${ }^{44}$ Da die Freiwilligkeit der Einwilligung in vielen Konstellationen problematisch ist und war, hat die DSGVO einige zusätzliche Erfordernisse formuliert, die bei der Beurteilung der Freiwilligkeit herangezogen werden können. Dazu gehören die Feststellung von Nachteilen für die betroffene Person, ein mögliches Un-

39 Zu letzterem Art.-29-Datenschutzgruppe 2018: 16.

40 BGHZ 117, 253 - Payback; BGH, Urt. v. 11.11.2009, Az. VIII ZR 12/08 - Happy Digits.

41 Z. B. Schantz/Wolff 2017: Rn. 492, Ingold 2020: Rn. 43; Art.-29-Datenschutzgruppe 2018: 16, Nebel 2018: Rn. 95.

42 Ernst 2018: Rn. 79.

43 Buchner/Kühling 2018: Art. 4 Nr. 11 DSGVO, Rn. 8 und Art. 7 DSGVO, Rn. 59.

44 Z. B. Erwägungsgrund 42 DSGVO, Heberlein 2018: Rn. 7. 
gleichgewicht zwischen betroffener Person und Verantwortlichem sowie die Koppelung der Einwilligung an andere Leistungen. Dabei sind in jedem Fall alle Umstände des Einzelfalls heranzuziehen. ${ }^{45}$

\subsection{Besonderheiten der Einwilligung von Kindern}

Ist die Einwilligung eines Kindes einzuholen, ist Art 8 DSGVO zu beachten. ${ }^{46}$ Nach diesem ist die Verarbeitung von personenbezogenen Daten im Rahmen von Diensten der Informationsgesellschaft, die Kindern direkt angeboten werden, dann rechtmäßig, wenn das Kind das sechzehnte Lebensjahr vollendet hat oder die Einwilligung oder Zustimmung des Sorgeberechtigten eingeholt wurde.

Lernplattformen sind Dienste der Informationsgesellschaft nach Art. 4 Nr. 25 DSGVO in Verbindung mit Art. 1 Nr. 1 lit. b der Informationsverfahrensrichtlinie. ${ }^{47}$ Sie sind elektronisch und auf individuellen Abruf des Empfängers erbrachte Dienstleistungen. Sie sind im Fernabsatz erbracht und erfolgen gegen Entgelt, entweder in Form einer Geldzahlung oder durch die Kommerzialisierung der Nutzerdaten. ${ }^{48}$

Der Dienst muss dem Kind direkt angeboten werden. Unstrittig sind Dienste, die sich durch die Art der Dienstleistung, die optische Gestaltung, kindgerechte Sprache und Anrede sowie Art der Werbung direkt an Kinder richten. ${ }^{49}$ Daneben sind auch solche Dienste erfasst, die sich sowohl an Erwachsene als auch an Kinder richten, indem sie keine konkrete Zielgruppe ansprechen („dual use“) ${ }^{50}$ oder minderjährige Nutzer durch ihre Allgemeinen Geschäftsbedingungen zulassen. ${ }^{51}$ Lernplattformen für Schüler richten sich naturgemäß an Kinder, aber auch an deren Sorgeberechtigten. Die Art der Dienstleistung bringt es zwingend mit sich, dass zumindest die Kinder diejenigen sind, die die Plattform nutzen, selbst wenn der Plattformbetreiber eher die Eltern anspricht. Daher gelten auch Lernplattformen für Schüler als direkt einem Kind angeboten.

45 Ausführlich zur Einwilligung Nebel 2020: 177 ff. mit weiteren Nachweisen.

46 Ausführlich zur Einwilligung bei Kindern auch Nebel 2020: $225 \mathrm{ff}$. mit weiteren Nachweisen.

47 Richtlinie 2015/1535/EU, ABl. EU 2015, L 241, 1.

48 Buchner/Kühling 2018: Art. 4 Nr. 25 DSGVO, Rn. 6.

49 Z. B. Buchner/Kühling 2018: Art. 8 DSGVO, Rn. 16, Klement 2019: Rn. 14.

50 Z. B. Buchner/Kühling 2018: Art. 8 DSGVO, Rn. 16, Frenzel 2018: Rn. 7, Klement 2019: Rn. 14.

51 So auch Kampert 2020: Rn. 9. 
Hat das Kind das 16. Lebensjahr noch nicht vollendet, so ist die Verarbeitung der personenbezogenen Daten des Kindes nur rechtmäßig, wenn und soweit der Träger der elterlichen Verantwortung - der Sorgeberechtigte, im Regelfall also die Eltern - eingewilligt oder zugestimmt haben. ${ }^{52} \mathrm{Da}$ die informationelle Selbstbestimmung ein höchstpersönliches Rechtsgut ist, muss die Einwilligung im Interesse des Kindes und Kindswohles erfolgen. ${ }^{53}$ Vom Sorgeberechtigten erfordert dies eine eigene, intensive Auseinandersetzung mit den Möglichkeiten und Risiken moderner Datenverarbeitungstechnologien. ${ }^{54}$ Nach Art. 8 Abs. 2 DSGVO hat der Verantwortliche unter Berücksichtigung der verfügbaren Technologien angemessene Anstrengungen zu unternehmen, um sich zu vergewissern, dass die Einwilligung durch den Sorgeberechtigten oder mit dessen Zustimmung gegeben wurde. Da ein Verstoß gegen Art. 8 DSGVO nach Art. 83 Abs. 5 lit. a DSGVO bußgeldbewehrt ist, kommt der Frage der Umsetzung des Art. 8 Abs. 2 DSGVO eine nicht zu unterschätzende Bedeutung zu.

In Deutschland hat das sich das sogenannte Double-Opt-in-Verfahren als Maßnahme zur Einholung der Einwilligung oder Zustimmung in der Praxis durchgesetzt. ${ }^{55}$ Dabei wird eine E-Mail an die E-Mail-Adresse der Sorgeberechtigten geschickt, mittels derer diese ihre Einwilligung oder Zustimmung erteilen können. Zwar besteht ein gewisses Missbrauchsrisiko durch das Kind, indem dieses eine zweite E-Mail-Adresse einrichtet, die den Anschein erweckt, dem Sorgeberechtigten zu gehören oder ohne deren Wissen die tatsächliche Mail-Adresse der Sorgeberechtigten nutzt. Formal wird es jedoch als ausreichend angesehen; die Umgehungsmöglichkeiten sind hinzunehmen, da dies zu verhindern der elterlichen Verantwortung obliegt und nicht den Verantwortlichen für die Datenverarbeitung. ${ }^{56}$

\section{Gestaltungsvorschläge und Fazit}

Lernplattformen sind eine wichtige Ergänzung sowohl im Rahmen des Schulalltags als auch außerhalb. Insbesondere wenn Kinder die Zielgruppe sind, ist jedoch ganz besonders auf den datenschutzkonformen Einsatz

52 Zur Abgrenzung von Einwilligung und Zustimmung z. B. Heckmann/Paschke 2018: Art. 8 DSGVO, Rn. 26 f.

53 Ausführlich Heckmann/Paschke 2018: Art. 8 DSGVO, Rn. 28 f.

54 AG Bad Hersfeld, Beschl. v. 15.5.2017, F 120/17 EASO.

55 Z. B. Schulz 2018: Art 8 DSGVO, Rn. 21, Heckmann/Paschke 2018: Art. 8 DSGVO, Rn. 37, Buchner/Kühling 2018: Art. 8 DSGVO, Rn. 24.

56 Z. B. Schulz 2018: Art 8 DSGVO, Rn. 21. 
und die Gestaltung der Plattformen Wert zu legen. Dies beginnt mit der Ermittlung der korrekten Rechtsgrundlage, die in der Praxis bisher nicht immer gelingt, da dies häufig mit Unsicherheiten und einigen rechtlichen Fallstricken behaftet ist. Der Beitrag hat erläutert, dass der Einsatz einer Lernplattform an einer Schule nicht auf Basis von Einwilligungen der Schüler bzw. deren Sorgeberechtigten erfolgen darf. Der zuständige Landesgesetzgeber muss vielmehr gesetzlich festlegen, dass eine Schule eine Lernplattform einsetzen darf. Er kann gemäß Art. 6 Abs. 2 und 3 DSGVO gleichzeitig spezifische Anforderungen für die Datenverarbeitung sowie sonstige Maßnahmen präziser bestimmen, um eine rechtmäßige und nach Treu und Glauben erfolgende Verarbeitung zu gewährleisten. Ebenso sollte unter den gleichen Voraussetzungen der Einsatz von Social Media an Schulen gesetzlich geregelt werden. Betreiber kommerzieller Plattformen müssen sich andererseits um eine wirksame Einwilligung der betroffenen Personen, im Falle von Kindern um die Einwilligung der Sorgeberechtigten, bemühen. Ein Rückgriff auf berechtigte Interessen oder die Verarbeitung zu Vertragszwecken ist häufig in diesem Fall nicht möglich. ${ }^{57}$

Für die organisatorische und technische Gestaltung von Lernplattformen sind weitere Faktoren wichtig, um eine datenschutzkonforme Nutzung zu gewährleisten. Hierzu zählen etwa die Schutzbedarfsanalyse der konkret zu verarbeitenden Daten und darauf aufbauende Ermittlung der zu ergreifenden Schutzmaßnahmen. ${ }^{58}$ Dazu gehört weiterhin die Datentrennung nach Nutzern und erhobenen Zwecken, um Risiken eines Datenmissbrauchs vorzubeugen. Wichtig ist außerdem die Umsetzung von Rollen- und Berechtigungskonzepten und Zugriffsrechten, welche vorab zu definieren sind, um sicherzustellen, dass nur berechtigte Personen und nur im notwendigen Umfang Zugriff auf die Daten haben. Insbesondere im schulischen Bereich ist darauf zu achten, dass Datenverarbeitung nur in dem Rahmen zulässig ist, wie das jeweilige Landes(schul)gesetz dies erlaubt. ${ }^{59}$ Bei der Implementierung der einzelnen Funktionalitäten der Plattform ist dies zu beachten. Weiterhin sind Löschkonzepte zu erstellen, um nicht mehr benötigte Daten rechtskonform löschen zu können. Es ist auf datenschutzfreundliche Voreinstellungen zu achten. Diese Pflicht ergibt sich bereits aus Art. 25 DSGVO. Schließlich ist die Anpassungsfähigkeit des Systems sicherzustellen, um auf rechtliche Änderungen reagieren zu

57 S. zu Vorschlägen für eine Fortentwicklung der DSGVO bezüglich des Datenschutzes von Kindern Roßnagel 2020a: $88 \mathrm{ff}$.

58 Hierfür bietet sich der Rückgriff auf das Standard-Datenschutzmodell der Datenschutzkonferenz an: DSK 2020.

59 DSK 2018: 5 . 
können. Für eine ausführliche Darstellung dieser und weiterer Gestaltungsvorschläge sei auf die Hinweise der Datenschutzkonferenz verwiesen. ${ }^{60}$

\section{Literatur}

Art.-29-Datenschutzgruppe (2018): Guidelines on consent. Working Paper 259 rev.01. Online verfügbar unter: https://ec.europa.eu/newsroom/article29/item-de tail.cfm?item_id=623051 (Abfrage am: 07.10.2020).

Buchner, Benedikt / Petri, Thomas (2018): Art. 6 DSGVO. In: Kühling, Jürgen / Buchner, Benedikt (Hg.): DS-GVO/BDSG, Kommentar (2). München: C.H.Beck.

Buchner, Benedikt / Kühling, Jürgen (2018): Art. 4 Nr.11 DSGVO sowie Art. 4 Nr. 25 DSGVO sowie Art. 7 DSGVO sowie Art. 8 DSGVO. In: Kühling, Jürgen / Buchner, Benedikt (Hg.): DS-GVO/BDSG, Kommentar (2). München: C.H.Beck.

Datenschutzkonferenz (DSK) (2018): Orientierungshilfe der Datenschutzbehörden für Online-Lernplattformen im Schulunterricht. Online verfügbar unter: https://www. datenschutzkonferenz-online.de/orientierungshilfen.html (Abfrage am: 07.10.2020).

Datenschutzkonferenz (DSK) (2020): Das Standard-Datenschutzmodell. Eine Methode zur Datenschutzberatung und -prüfung auf der Basis einheitlicher Gewährleistungsziele. Version 2.0b. Online verfügbar unter: https://www.datenschutzzentrum.de/sd $\mathrm{m} /$ (Abfrage am: 07.10.2020).

Ernst, Stefan (2018): Art. 4 DSGVO. In: Paal, Boris / Pauly, Daniel (Hg.): Datenschutz-Grundverordnung, Bundesdatenschutzgesetz, Kommentar (2). München: C.H.Beck.

Frenzel, Eike Michael (2018): Art. 8 DSGVO. In: Paal, Boris / Pauly, Daniel (Hg.): Datenschutz-Grundverordnung, Bundesdatenschutzgesetz, Kommentar (2). München: C.H.Beck.

Gierschmann, Sibylle (2018): Gestaltungsmöglichkeiten bei Verwendung von personenbezogenen Daten in der Werbung. In: MultiMedia und Recht (1), S. 7-12.

Heberlein, Johanna (2018): Art. 6 DSGVO. In: Ehmann, Eugen / Selmayr, Martin (Hg.): DS-GVO, Kommentar (2). München: C.H.Beck.

Heckmann, Dirk / Paschke, Anne (2018): Art. 7 DSGVO sowie Art. 8 DSGVO. In: Ehmann, Eugen / Selmayr, Martin (Hg.): DS-GVO, Kommentar (2). München: C.H.Beck.

Helfrich, Marcus (2020): Art. 21 DSGVO. In: Sydow, Gernot (Hg.): Europäische Datenschutzgrundverordnung (2). Baden-Baden: Nomos.

60 DSK 2018: $12 \mathrm{ff}$. 
Hornung, Gerrit (2015): Datenschutzrechtliche Aspekte der Social Media. In: Hornung, Gerrit / Müller-Terpitz, Ralf (Hg.): Rechtshandbuch Social Media. Berlin, Heidelberg: Springer, S. 79-130.

Ingold, Albert (2020): Art. 7 DSGVO. In: Sydow, Gernot (Hg.): Europäische Datenschutzgrundverordnung (2). Baden-Baden: Nomos.

Jandt, Silke / Schaar, Peter, / Schulz, Wolfgang (2013): \$13 TMG. In: Roßnagel, Alexander (Hg.): Recht der Telemediendienste. München: C.H.Beck.

Kampert, David (2020): Art. 8 DSGVO. In: Sydow, Gernot (Hg.): Europäische Datenschutzgrundverordnung (2). Baden-Baden: Nomos.

Karg, Moritz / Fahl, Constantin (2011): Rechtsgrundlagen für den Datenschutz in sozialen Netzwerken. In: Kommunikation \& Recht (7/8), S. 453-458.

Karg, Moritz / Thomsen, Sven (2012): Tracking und Analyse durch Facebook. In: Datenschutz und Datensicherheit 36 (10), S. 729-736.

Klement, Jan Henrik (2019): Art. 8 DSGVO. In: Simitis, Spiros / Hornung, Gerrit / Spiecker gen. Döhmann, Indra (Hg.): Datenschutzrecht, Kommentar. Baden-Baden: Nomos.

Nebel, Maxi (2018): Erlaubnis zur Datenverarbeitung (\$3). In: Roßnagel, Alexander (Hg.): Das neue Datenschutzrecht, Nomos: Baden-Baden, S. 104-115.

Nebel, Maxi (2020): Persönlichkeitsschutz in Social Networks, Technische Unterstützung eines grundrechtskonformen Angebots von Social Networks. Wiesbaden: Springer.

Profit (2018): $\int 83$ HessSchulG Erhebung und Verarbeitung personenbezogener Daten. In: Köller / Achilles: Hessisches Schulgesetz. Wiesbaden: Kommunal- und Schulverlag.

Ratzsch, Jörg (2019): Studie: Viele Kinder nutzen Youtube zum Lernen für die Schule. Online verfügbar unter: https://heise.de/- 4438484 (Abfrage am: 07.10.2020).

Roßnagel, Alexander (2019): Art. 2 DSGVO sowie Art. 6 Abs. 1 DSGVO sowie Art 6 Abs. 2 DSGVO. In: Simitis, Spiros / Hornung, Gerrit / Spiecker gen. Döhmann, Indra (Hg.): Datenschutzrecht, Kommentar. Baden-Baden: Nomos.

Roßnagel, Alexander / Geminn, Christian / Johannes, Paul C. (2019): DatenschutzFolgenabschätzung im Zuge der Gesetzgebung. In: Zeitschrift für Datenschutz (10), S. 435-440.

Roßnagel, Alexander (2020a): Der Datenschutz von Kindern in der DS-GVO. In: Zeitschrift für Datenschutz (2), S. 88-92.

Roßnagel, Alexander (2020b): Datenschutz im E-Learning. In: Zeitschrift für Datenschutz (6), S. 296-302.

Sassenberg, Elke (2019): Datenschutz in Schule und Schulverwaltung (\$ 24). In: Specht, Louisa / Mantz, Reto (Hg.): Handbuch Europäisches und deutsches Datenschutzrecht. München: C.H.Beck, S. 672-698.

Schaller, Fabian: Datenschutz im öffentlichen Bereich (\$ 7). In: Roßnagel, Alexander (Hg.): Das neue Datenschutzrecht. Baden-Baden: Nomos, S. 270-281. 
Schantz, Peter (2019): Art. 6 Abs. 1 DSGVO. In: Simitis, Spiros / Hornung, Gerrit / Spiecker gen. Döhmann, Indra (Hg.): Datenschutzrecht, Kommentar. Baden-Baden: Nomos.

Schantz, Peter / Wolff, Heinrich A. (2017): Das neue Datenschutzrecht. München: C.H.Beck.

Schulz, Sebastian (2018): Art. 6 DSGVO sowie Art. 8 DSGVO sowie Art. 21 DSGVO. In: Gola, Peter (Hg.): Datenschutz-Grundverordnung, Kommentar. 2. Aufl. München: C.H.Beck. 
